\title{
Socioeconomic status: a disease modifier of chronic rhinosinusitis?*
}

\author{
Shaun J. Kilty ${ }^{1}$, J. Ted McDonald ${ }^{2}$, Stephanie Johnson ${ }^{1}$, Dakheelallah Al-Mutairi ${ }^{1}$ \\ Department of Otolaryngology-Head and Neck Surgery, Ottawa Hospital, \\ University of Ottawa, Ottawa, ON, Canada \\ 2 Department of Economics, University of New Brunswick, Fredericton, NB, Canada
}

SUMMARY

Introduction: The Lund-MacKay score (LMS) correlates poorly with chronic rhinosinusitis (CRS) symptom severity. Patients with CRS also tend to report relatively lower levels of mental wellbeing. Our purpose was to determine if there is a correlation between socio-economic status (SES) and CRS severity as measured by the LMS, and if there is an association between depression symptoms and the severity of CRS using the LMS.

Methods: A total of 127 patients diagnosed with CRS were prospectively recruited and assessed with a sinonasal assessment questionnaire (SNAQ-11), and the Patient Health Questionnaire (PHQ-9) for depression. Each patient's education level, family income, and smoking behavior were determined. The sinus CT scan was scored using the LMS. The data were analyzed using ordinary least squares (OLS) regression techniques.

Results: Having a highschool education or less was associated with higher SNAQ-11 scores while being a daily smoker was associated with higher SNAQ-11 scores. There was no significant relationship between educational attainment, financial income or daily smoking and sinus CT score. Including depression scores in the SNAQ-11, regression equations indicated a significant and positive relationship between depression severity and SNAQ-11 score. CRS with polyps was negatively associated with SNAQ-11 scores but, as expected, it was positively associated with a higher LMS.

Conclusions: Lower SES status is a negative modifying factor of subjective CRS severity but it has no impact on the LMS. Depression symptoms are associated with increased subjective CRS severity but they have no effect on the LMS. How SES and depression impact on a patient's self-reported disease severity requires further study.

Key words: chronic rhinosinusitis, socioeconomic status, sinusitis symptoms, depression, Lund-McKay score, computed tomography scan, sinonasal assessment questionnaire (SNAQ-11).

\section{INTRODUCTION}

Chronic rhinosinusitis (CRS) is a common inflammatory disease that is multifactorial in etiology. It is one of the three most common chronic diseases in North America ${ }^{(1)}$. In Canada, it has an estimated prevalence of $5.7 \%{ }^{(2)}$. CRS is a cause of significant morbidity with respondents reporting poorer scores in measures of bodily pain and social functioning when compared to patients with CHF, angina, COPD and back pain ${ }^{(3)}$. Results from a recent population survey suggested that individuals who report having CRS will also have lower levels of mental and physical health ${ }^{(4)}$.
To date, most studies have shown that there is little correlation between the subjective symptoms of a patient with CRS and computed tomography (CT) scores as measured by the LundMacKay score (LMS) ${ }^{(5-7)}$. Given this disconnect, the factors that affect a patient's self-reported symptom severity for CRS have yet to be defined. Our purpose was to determine if there is a statistically significant correlation between socio-economic status (SES) and CRS CT severity as measured by the LMS and if there is an association between depression symptoms and the objective CT (LMS) disease severity in patients with CRS. 


\section{METHODS}

\section{Study design}

This prospective study was approved by the Ottawa Hospital Research Ethics Board (OHREB). Patients diagnosed with CRS with or without polyps using the criteria of a published guideline ${ }^{(1)}$ in a tertiary rhinology clinic were recruited. They completed the sinonasal assessment questionnaire (SNAQ-11) and a validated questionnaire for depression using the Patient Health Questionnaire (PHQ-9). The SNAQ-11 is a specific CRS symptom and quality of life measure that has been validated using the sinonasal outcome test (SNOT-20) ${ }^{(8)}$. It has a minimal time requirement and it has been found to be reflective of patient symptoms both before and after treatment ${ }^{(8)}$. The PHQ-9 is a validated clinical screening questionnaire for the detection of depressive symptoms and depression ${ }^{(9)}$. It too has a minimal time requirement. A CT scan of the paranasal sinuses was completed as part of their evaluation and was scored using the Lund-MacKay system. In addition, we collected information on each patient's highest level of educational attainment, family income, smoking behavior as well as demographic information including age and gender.

\section{Statistical analysis}

We then estimated a series of regression equations in which the dependent variable was specified as the sinus severity score (measured both by SNAQ-11 score and LMS). In the first set of regressions, we included independent variables for gender, age, age-squared, educational attainment (high school or less and postsecondary education), and whether the patient was a daily smoker at the time of the interview. These basic regression results (no depression, no polyps) for SNAQ-11 and LMS allowed us to establish the difference in SES. We then included depression scores (PHQ-9) and polyps for each measure in a second set of regressions to gauge the extent to which these measures moderate the relationships between severity and SES. Specifically, we included a known correlate of SNAQ-11 (depression) to see if it affects LMS, and a known risk factor for LMS (polyps) to see if it affects SNAQ-11 scores. We estimate the main models both with and without depression score given that depression itself has been shown to be correlated with education level and other factors ${ }^{(10-12)}$, and the inclusion of this co-morbid condition may reduce the magnitude and significance of our key variables of interest. Given the roughly

Table 1. Characteristics of the study cohort.

\begin{tabular}{|c|c|c|c|}
\hline & $\begin{array}{c}\text { Average value/proportion of } \\
\text { the sample }\end{array}$ & $\begin{array}{c}\text { Self-reported sinus score } \\
\text { (SNAQ-11) }\end{array}$ & Lund-Mackay score \\
\hline All $(\mathrm{n}=127)$ & 1 & 39.0 & 14.2 \\
\hline Depression Severity (PHQ-9 score) & & 34.7 & 14.7 \\
\hline Minimal or none $(0-4)$ & 0.53 & & \\
\hline Mild (5-9) & 0.24 & 41.7 & 13.4 \\
\hline Moderate (10-14) & 0.15 & 42.9 & 14.0 \\
\hline Moderate Severe or Severe (15-27) & 0.08 & 53.0 & 13.2 \\
\hline Age (years) & 50.9 & & \\
\hline Male & 0.51 & 38.5 & 14.8 \\
\hline Female & 0.49 & 39.4 & 13.5 \\
\hline High school or less & 0.24 & 45.0 & 15.0 \\
\hline Non-university post-secondary & 0.37 & 38.4 & 14.4 \\
\hline University Degree & 0.39 & 35.6 & 13.5 \\
\hline Income $<\$ 40,000$ & 0.23 & 44.1 & 13.1 \\
\hline Income $\$ 40-80,000$ & 0.30 & 35.2 & 15.4 \\
\hline Income $>\$ 80,000$ & 0.47 & 38.4 & 14.0 \\
\hline Smokes daily & 0.16 & 49.3 & 13.0 \\
\hline Does not smoke daily & 0.84 & 36.9 & 14.4 \\
\hline Has polyps & 0.62 & 36.3 & 17.3 \\
\hline No polyps & 0.38 & 43.4 & 9.2 \\
\hline
\end{tabular}


Table 2. Results of analyses of patient characteristics versus SNAQ-11 scores and LMS.

\begin{tabular}{|c|c|c|c|c|c|c|}
\hline & \multicolumn{3}{|c|}{ SNAQ-11 } & \multicolumn{3}{|c|}{ Lund-MacKay Score (LMS) } \\
\hline & OLS Coef. & P-value & $95 \% \mathrm{CI}$ & OLS Coef. & P-value & $95 \% \mathrm{CI}$ \\
\hline Male & -0.99 & 0.75 & {$[-7.1,5.1]$} & 0.73 & 0.50 & {$[-1.4,2.9]$} \\
\hline Age & $1.92 *$ & 0.01 & {$[.54,3.3]$} & 0.03 & 0.89 & {$[-.45, .52]$} \\
\hline Age-squared & $-0.02 *$ & 0.00 & {$[-.03,-.01]$} & 0.00 & 0.99 & {$[-.00, .00]$} \\
\hline Smokes daily & 8.16 & 0.06 & {$[-.49,16.8]$} & -1.80 & 0.24 & {$[-4.9,1.3]$} \\
\hline High school or less & $6.89 *$ & 0.05 & {$[.08,13.7]$} & 0.83 & 0.50 & {$[-1.6,3.2]$} \\
\hline Post-secondary & -- & & & -- & & \\
\hline Constant & -0.99 & 0.75 & {$[-40,30]$} & 0.73 & 0.50 & {$[-.06,25]$} \\
\hline $\mathrm{N}$ & 127 & & & 127 & & \\
\hline adj-R2 & 0.13 & & & -0.02 & & \\
\hline
\end{tabular}

* denotes significantly different from zero at the $5 \%$ level of significance.

continuous nature of the dependent variable, we employed ordinary least squares (OLS) regression techniques. As a check of the robustness of our results, we repeated the analysis using family income as our measure of SES instead of educational attainment. (Including both education and income controls did not yield any additional insights.)

\section{RESULTS}

The baseline characteristics of the study cohort and sinus scores by characteristic are presented in Table 1. In this study, 127 patients were enrolled with a mean age of 50 years old and $51 \%$ of the cohort was male. Just over $60 \%$ of patients had CRS with polyps and only $16 \%$ of the cohort were daily smokers. The mean LMS was 14. Minimal or no depressive symptoms were present in $53 \%$ of the patients but nearly onequarter had symptoms of at least a mild depression. The distribution of patients was fairly equal for educational attainment whereas for financial income, the largest proportion of patients was in the highest income bracket.

Power calculations were completed for SNAQ-11 and LundMacKay scores. For the SNAQ-11, with a total sample of $\mathrm{n}=$ 127 and an alpha of 5\%, detecting a 10\% increase in sinus score has a power stat of 0.71 on a two sided test and 0.81 on a onesided test. For the Lund-MacKay score, with the same parameters, detecting a $10 \%$ increase in the CT score has a power stat of 0.69 on a two sided test and 0.79 on a one sided test. The simple correlation coefficient between LMS and SNAQ-11 is -0.08 and is not significantly different from zero $(p=0.652)$.

As can be seen in Table 2, having an educational level of highschool or less is significantly associated with self-reported sinus symptom score and it is nearly 7 points higher than for a person with post-secondary education (coef $6.89, \mathrm{p}=0.05$ ). As well, daily smokers have a self-reported score that is on average 8 points higher than what is the case for non-smokers (coef $8.16, \mathrm{p}=0.06$ ), but this was not statistically significant. The SNAQ-11 score increases with age (coef 1.92, $\mathrm{p}=0.01$ ) but at a decreasing rate since the coefficient on the squared-age term is negative (coef $-0.02, p=0.00$ ). In contrast, there is no significant relationship between the Lund-MacKay score and any of the aforementioned factors including gender.

In Table 3, the depression score is positively and significantly related to self-reported sinus symptom score, with a coefficient that implies a roughly one-point increase in the PHQ-9 is associated with a one-point increase in the SNAQ-11 (coef 0.61, p $=0.04)$. The inclusion of depression score reduces the magnitude and level of significance of education and daily smoking, suggesting that depression among sinusitis patients is positively correlated with postsecondary education and positively correlated with daily smoking. In contrast, there is no significant positive relationship found between depression score and CT score, with a negative coefficient that is small and poorly determined (coef $-0.01, p=0.85$ ). The presence of polyps was negatively but significantly correlated with sinus symptoms suggesting that patients with polyps rated their disease severity to be about 7 points lower than patients without polyps (coef $-7.13, \mathrm{p}=0.03$ ). However, the presence of polyps was positively and significantly correlated to higher Lund-MacKay scores, with patients with polyps having scans that are 8 points higher than patients without polyps (coef $=8.07, \mathrm{p}=0.00)$.

Finally, repeating the analysis using a set of indicators for family income (<\$20k, \$20 - 40k, \$40 - 80k, \$80k+) instead of education controls yielded similar conclusions: lower family income is related to higher self-reported sinus disease symptom severity but has no effect on sinus CT score. 
Table 3. Results of analyses of depression and presence of polyps on SNAQ-11 scores and LMS.

\begin{tabular}{|c|c|c|c|c|c|c|}
\hline & \multicolumn{3}{|c|}{ SNAQ-11 } & \multicolumn{3}{|c|}{ Lund-MacKay Score (LMS) } \\
\hline & OLS Coef. & P-value & $95 \% \mathrm{CI}$ & OLS Coef. & P-value & $95 \% \mathrm{CI}$ \\
\hline Male & 0.86 & 0.78 & {$[-5.2,6.9]$} & 0.39 & 0.65 & {$[-1.3,2.1]$} \\
\hline Age & $1.94^{*}$ & 0.01 & {$[.59,3.3]$} & -0.14 & 0.46 & {$[-.52, .23]$} \\
\hline Age-squared & $-0.02 *$ & 0.00 & {$[-.03,-.01]$} & 0.00 & 0.35 & {$[-.00, .01]$} \\
\hline Smokes daily & 5.97 & 0.17 & {$[-2.6,14.6]$} & -0.39 & 0.75 & {$[-2.8,2.0]$} \\
\hline High school or less & 6.25 & 0.07 & {$[-.47,13.0]$} & 0.11 & 0.91 & {$[-1.8,2.0]$} \\
\hline Post-secondary & -- & & & -- & & \\
\hline Depression score & $0.61 *$ & 0.04 & {$[.04,1.2]$} & -0.01 & 0.85 & {$[-.17, .14]$} \\
\hline Polyps & $-7.13^{*}$ & 0.03 & {$[-13.4,-.84]$} & $8.07 *$ & 0.00 & {$[6.3,9.8]$} \\
\hline Constant & -7.02 & 0.68 & {$[-41,27]$} & $11.53^{*}$ & 0.02 & {$[2.1,21]$} \\
\hline $\mathrm{N}$ & 127 & & & 127 & & \\
\hline $\operatorname{adj}-R 2$ & 0.19 & & & 0.40 & & \\
\hline
\end{tabular}

* denotes significantly different from zero at the $5 \%$ level of significance.

\section{DISCUSSION}

The symptomatology of individuals with CRS is variable. Objective measures of CRS such as CT score do not always reflect individual subjective evaluations of CRS severity. The factors that may influence this relationship remain to be established. In this study, patients who had polyps reported lower symptom scores than patients without polyps (coef -7.13, $\mathrm{p}=0.03$ ). However, as expected, patients with CRS with polyps did have higher Lund-MacKay scores. As such, our data do not support the presence of polyps as a significant determinant of subjective CRS symptomatology. Therefore, from these results we confirm a common clinical presumption, that the presence of polyps is significantly associated with a higher disease burden on the CT (coef 8.07, $\mathrm{p}=0.00$ ).

Furthermore, our data indicate that in this cohort, individuals with lower levels of SES report significantly higher subjective levels of CRS symptomatology than individuals of higher SES while their CT scans do not demonstrate increasing disease severity. How lower SES status may lead to this effect is not clear. However, SES has been shown to impact subjective measures of pain, with individuals of lower SES reporting increasing pain severity with objectively equal pain levels to individuals of higher SES ${ }^{(13)}$. Individuals of lower SES are similarly more likely to feel a greater degree of disability due to their pain ${ }^{(13)}$. Our findings may therefore reflect the contribution of a SES factor to the social component in the biopsychosocial model of disease ${ }^{(14)}$. Further study is needed to better understand this relationship of low SES and increased selfreported disease severity in CRS.

Similarly, in this study participants with higher depression scores rated their disease to be subjectively more severe although the objective radiologic measure of their disease did not support this relationship. In patients with rheumatoid arthritis, a similar relationship has been found with increasing levels of depression being associated with increased subjective reports of pain, lesser coping abilities and increased disability (15). Therefore, a patient's experience with CRS may affect their psychological assessment of its physical effects and manifestations. In the end, this interaction of the physical symptomatology and psychology in patients with CRS is not as of yet understood and it is a subject to be further studied.

\section{Study limitations}

A potential limitation of this study is that since the incidence of post-secondary education (higher SES) among patients in this study is higher than in the overall Canadian population, it may indicate recruitment bias. Given that our patient catchment area is metropolitan Ottawa, the average level of educational attainment is higher than that of the overall population. However, even in the setting of universal healthcare in Canada, SES has been shown with other medical diagnoses to affect referral for specialist services from a primary care practitioner (PCP) ${ }^{(16-19)}$. These studies have demonstrated that in general, individuals of higher SES more commonly receive a specialist referral than individuals of low SES. Therefore, the higher SES incidence of this study does not necessarily represent a bias in recruitment for this study, but rather the actual referral patterns from PCPs to the clinic for patients with CRS. Therefore, not only may SES affect both the incidence of diagnosis and self-reported severity, but SES may also affect referral patterns of patients with CRS from PCPs to Otolaryngologists. This last point however, has not been investigated in this study and requires further research.

The employment of a control group may have served to better understand the studied CRS population and to determine if a true bias existed in the referral pattern of patients with CRS. A further limitation of this study is the use of the SNAQ-11 as an outcome measure. The SNAQ-11 is a validated easy to use tool for patient self-reporting of the symptoms of CRS ${ }^{(8)}$. However, it is not used as frequently as other measures such as the SNOT-22, which limits the comparison of results that can be made with this study and others using the SNOT-22. 


\section{CONCLUSIONS}

There is poor correlation between subjective and objective measures of CRS. SES status is a modifying factor of self-rated CRS severity however it was not related to CT scan disease severity. Patients with at least mild depressive symptoms are likely to report a greater severity in their subjective CRS symptoms without an associated increase in their LMS. Further study is required to understand these findings and the interaction of SES and depression on CRS.

\section{REFERENCES}

1. Benninger, Ferguson BJ, Hadley JA, et al. Adult chronic rhinosinusitis: definitions, diagnosis, epidemiology, and pathophysiology. Otolaryngol Head Neck Surg. 2003; 129 (Suppl): S1-32.

2. Chen Y, Dales R, Lin M. The epidemiology of chronic rhinosinusitis in Canadians. Laryngoscope. 2003; 113: 1199-1205.

3. Gliklich RE, Metson R. The health impact of chronic sinusitis in patients seeking otolaryngologic care. Otolaryngol Head Neck Surg. 1995; 113: 104-109.

4. MacDonald KI, McNally JD, Massoud E. The health and resource utilization of Canadians with chronic rhinosinusitis. Laryngoscope. 2009; 119(1): 184-189.

5. Stewart MG, Sicard MW, Piccirillo JF, Diaz-Marchan PJ. Severity staging in chronic sinusitis: are CT scan findings related to patient symptoms? Am J Rhinol. 1999; 13: 161-167.

6. Gaskins RE. A surgical staging system for chronic sinusitis. Am J Rhinol. 1992; 6: 5-12.

7. Bhattacharyya T, Piccirillo J, Wippold FJ. Relationship between patient-based descriptions of sinusitis and paranasal sinus CT. Arch Otolaryngol Head Neck Surg. 1997; 123: 1189-1192.

8. Fahmy FF, McCombe A, Mckiernan DC. Sino nasal assessment questionnaire, a patient focused, rhinosinusitis specific outcome measure. Rhinology. 2002; 40: 195-197.

9. Kendel F, Wirtz M, Dunkel A, et al. Screening for depression: Rasch analysis of the dimensional structure of the PHQ-9 and the HADS-D. J Affect Disord. 2010; 122: 241-246.

10. Miech RA and Shanahan MJ. Socioeconomic status and depression over the life course. Journal of Health and Social Behavior. 2000; 41: 162-176.
11. Everson SA, Maty SC, Lynch JW, Kaplan GA. Epidemiologic evidence for the relation between socioeconomic status and depression, obesity, and diabetes. J Psychosom Res. 2002; 53: 891-895.

12. Lorant $\mathrm{V}$, Deliège $\mathrm{D}$, Eaton $\mathrm{W}$, et al. Socioeconomic Inequalities in Depression: A Meta-Analysis. Am. J. Epidemiol. 2003; 157: 98-112.

13. Dorner TE, Muckenhuber J, Stronegger WJ, et al. The impact of socio-economic status on pain and the perception of disability due to pain. Eur J Pain. 2011; 15: 103-109.

14. Engel GL. The need for a new medical model: a challenge for biomedicine. Science. 1977; 196: 129-136.

15. Zyrianova Y, Kelly BD, Sheehan J, et al. The psychological impact of arthritis: the effects of illness perception and coping. Ir J Med Sci. 2011; 180: 203-210.

16. Haider A, Mamdani M, Shaw JC, Alter DA, Shear NH. Socioeconomic status influences care of patients with acne in Ontario, Canada. J Am Acad Dermatol. 2006; 54: 331-335.

17. Dunlop S, Coyte PC, McIsaac W. Socio-economic status and the utilization of physicians' services: results from the Canadian National Population Health Survey. Soc Sci Med. 2000; 51: 123-133.

18. Chan BT, Austin PC. Patient, physician, and community factors affecting referrals to specialists in Ontario, Canada: a populationbased, multi-level modeling approach. Med Care. 2003; 41: 500-511.

19. Glazier RH, Agha MM, Moineddin R, Sibley LM. Universal health insurance and equity in primary care and specialist office visits: a population-based study. Ann Fam Med. 2009; 7: 396-405.

Dr. Shaun Kilty

Department of Otolaryngology-Head and Neck Surgery

Ottawa Hospital, Civic Campus

Parkdale Clinic, Room 242

1053 Carling Ave., Ottawa, ON

Canada

Tel: +1-(613)-761 4030

Fax: +1-(613)-729 2412

E-mail: shaunkilty@hotmail.com

\section{ADVERTISEMENT}

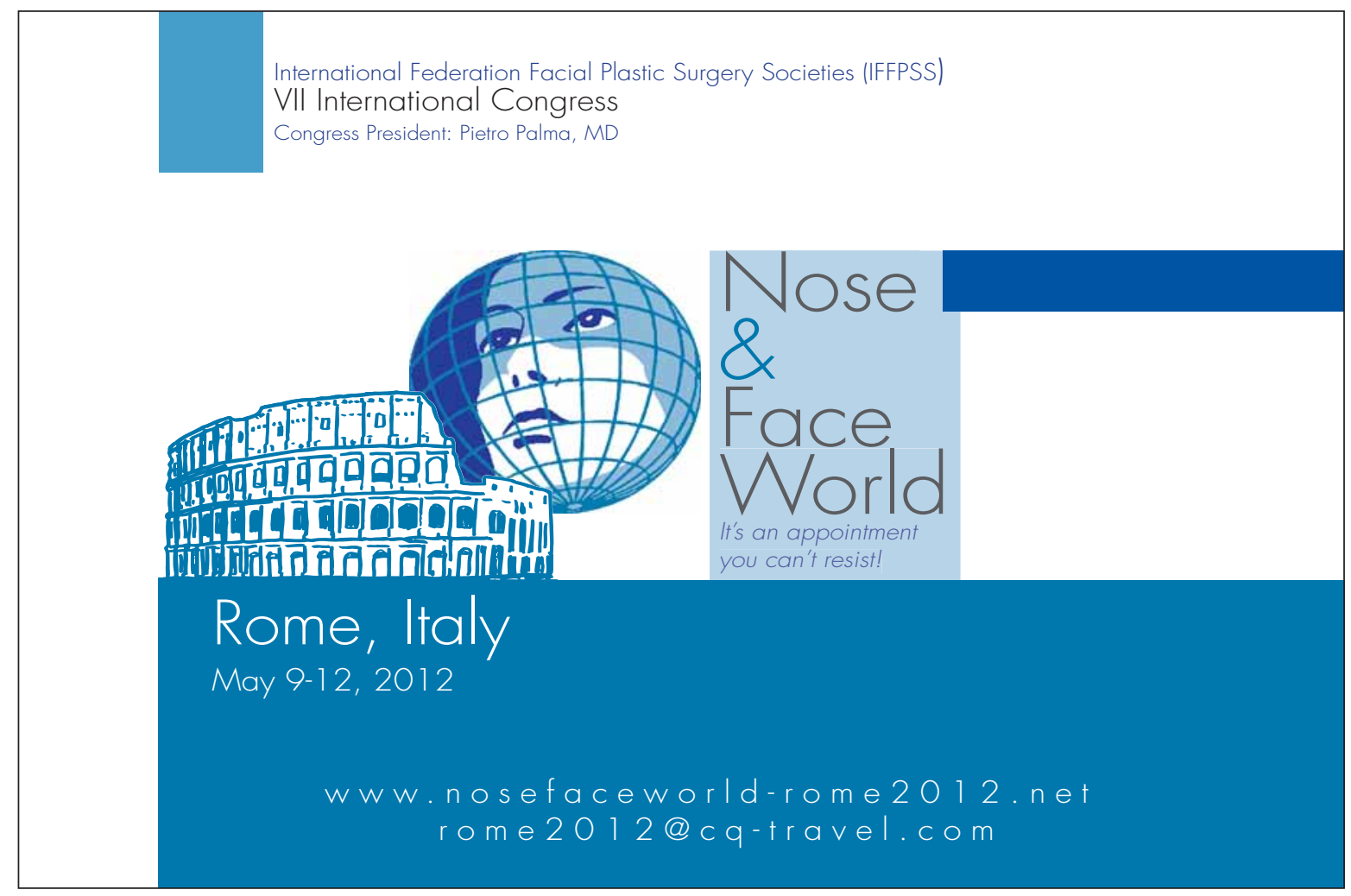

